\title{
Familial Hypertrophic Cardiomyopathy. Genetic Characterization
}

\author{
Adriana Paula Tirone, Edmundo Arteaga
}

São Paulo, SP - Brazil

This article aims to show clearly, especially to cardiologists, the currently recognized genetic mutations responsible for hypertrophic cardiomyopathy (HCM). $\mathrm{HCM}$ is a polygenic disease with major clinical heterogeneity and diagnostic and prognostic implications peculiar to each mutation. Most recently, the search for new mutations has increased interest in clinical and laboratorial studies of this disease.

$\mathrm{HCM}$ is a complex cardiac disease with unique physiopathological characteristics and great diversity of morphological, functional and clinical features ${ }^{1}$. It is a primary cardiac disorder, in which the basic diagnostic characteristic is left ventricle hypertrophy $(\mathrm{LVH})$ without ventricular dilation, in the absence of any heart or systemic disease that can lead to myocardial hypertrophy ${ }^{2,3}$.

Prevalence of HCM in the general population is about $0.2 \%$ ( 1 in 500), greater than thought in the past. It is probably the most common genetically transmitted cardiovascular disease. Annual mortality for HCM patients is 3\% to $4 \%$ and over $6 \%$ in children, i.e., substantially greater than in nonselected patients $(0.5 \% \text { to } 1 \%)^{1,4}$.

Clinical course varies from totally asymptomatic patients throughout life to those with major heart failure symptoms and even sudden death ${ }^{2,4,5}$.

In more than $50 \%$ of the cases, $\mathrm{HCM}$ is an autosomal dominant genetic disease and, in the remaining cases, we still do not have a defined etiology, which can be also genetic but with mutations not yet recognized. Up to now, alterations in seven genes codifying proteins of the sarcomere have been identified: the cardiac b-myosin heavy chain ( $\beta$ MYHC) gene; the cardiac troponin T (cTnT) gene; the a-tropomyosin gene; the myosin-binding protein$\mathrm{C}$ (MyBP-C) gene; the cardiac troponin I gene; and the regulatory and essential myosin light chain gene ${ }^{5,6}$.

The cardiac $\beta$-myosin heavy chain gene is located in the chromosome 14 (locus q1), where 40 more mutations causing the disease have been identified, most of them

Instituto do Coração do Hospital das Clínicas - FMUSP

Mailing address: Adriana Paula Tirone - Incor - Av. Dr. Enéas C. Aguiar, $44-$ 05403-000 - São Paulo, SP - Brazil occurring by substitution of DNA nucleotides, resulting in the change of one amino acid in the protein sequence ${ }^{7,8}$. This change occurs in $30 \%$ to $40 \%$ of familial HCM cases, and there are mutations with high, intermediate and low risk of sudden death (SD) ${ }^{2,4,8}$. The Arg403Gln (where arginine was substituted by glycine in position 403), Arg453Cys and Arg719Trp mutations are considered malignant ${ }^{4,7,8}$. Phenotypes associated with Arg403Gln are seen in many families and are characterized as of high penetrance, high SD incidence and severe hypertrophy ${ }^{2,7,8}$. Studies ${ }^{9}$ on mortality in two families with the mutation Arg403Gln were carried out in which 21 out of 44 affected individuals died (nine with SD), 33 years being the mean age. Marian and Roberts ${ }^{2,4}$ identified two families with this same mutation, in which 11 out of 20 affected individuals died (9 with SD) and the mean age was also 33 years.

The second mutation with a high incidence of SD is Arg719Trp, described in four families composed of 61 individuals, of which 34 died ( 22 by SD) and the mean age was 38 years. Arg453Cys is another malignant mutation, seen in a family in which 9 out of 13 affected individuals died (six with SD), 30 years being the mean age ${ }^{2,4}$.

The Glu930Lys and Arg249GIn mutations are associated with an intermediate risk of $\mathrm{SD}^{2}$. Marian and Roberts ${ }^{2,4}$ described a family composed of 16 individuals with Glu930Lys mutation, in which two died at the ages of 14 and 16 years, and another underwent heart transplantation at the age of 58 years.

The Leu908Val, Gly256Glu, Val606Met, Fhe513Cys mutations are associated with benign prognostic and normal survival ${ }^{4,7}$. The Leu908Val mutation has been described in a big family composed of 46 affected individuals, from which only two died ${ }^{2,4}$. The Gly256Glu mutation occurred in a family of 39 affected individuals and the mortality rate was $2 \%$ at the age of 50 years. The Val606Met mutation is also associated with benign prognostic survival in four families. Watkins et al $^{9}$ showed this mutation in three small families composed of 18 affected individuals, from which only one died. Marian et $\mathrm{al}^{4}$ identified this mutation in one family of 12 affected individuals, from which only one died. Fananapazir et $\mathrm{al}^{10}$, however, showed a family with this mutation with severe hypertrophy and high incidence of SD.

Cardiac troponin $\mathrm{T}$ gene is located in chromosome 1 (locus q3), where eight mutations have been identified so 
far. It happens in about $15 \%$ of the cases. The main characteristic of the mutations in this gene is that they cause discrete hypertrophy, but with a high incidence of SD in young patients $(<30 \text { years old })^{2,11}$.

Alpha-tropomyosin gene is located in chromosome 15 (locus q2) and two mutations have been observed so far. It seems to occur in 5\% of the HCM cases and is associated with normal survival ${ }^{2,11}$.

Myosin-binding protein-C gene is located in chromosome 11 (locus p 11.2) ${ }^{5}$. So far, 14 mutations have been identified, four of which result from nucleotide changes and eight are truncated mutations ${ }^{5,12}$. It occurs in $15 \%$ of the cases and its main characteristic is low penetrance up to the $5^{\text {th }}$ decade of life with moderate hypertrophy and satisfactory survival ${ }^{6,12}$.

Regulatory and essential myosin light chain genes were found in $1996^{9}$. The regulator gene (MYL2) is found in chromosome 12 (locus q23 and q24.3) and the essential gene (MYL3) is found in chromosome $3 \mathrm{p}^{5,13}$. So far, there are four mutations described for these genes, which show mild hypertrophy and good prognosis ${ }^{13,14}$.

For troponin I gene, there are apparently four mutations still under study.

There is evidence suggesting that other genetic factors influence hypertrophy ${ }^{2,15,16}$. A deletion/insertion polymorphism of the angiotensin-converting enzyme (ACE) gene, characterized by D and I alleles resulting in three genotypes II, ID and DD, is associated with differences in the plasmatic levels of ACE and its activity ${ }^{17,18}$. DD genotype is related to an increase in ACE levels and, in several reports, it seems to be a risk factor for myocardial infarction, dilated cardiomyopathy, postangioplasty restenosis, and $\mathrm{HCM}^{8,18}$. It was initially observed that the frequency of the $\mathrm{D}$ allele increased only in families with high incidence of $\mathrm{SD}^{2,4,8}$, and its relation with the increase in cardiac hypertrophy was later shown ${ }^{8,15,19}$.

A second gene of the renin-angiotensin system associated with cardiac disease is the angiotensinogen. The polymorphism occurs in the position 235 where threonine is replaced by methionine (M/T). TT genotype seems to be related to greater risk of myocardial hypertrophy. Ishanov et $\mathrm{al}^{20}$, studying a Japanese subpopulation, observed that hypertrophy was greater in individuals having one or two D alleles, in addition to T allele contribution, which may constitute a marker for a critical gene in HCM or in myocardial hypertrophy.

Identifying mutations responsible for HCM leads to an important diagnostic arsenal. The present diagnosis is made by an echocardiogram showing $\mathrm{LVH}$, but this is not evident in many cases until puberty. However, a genetic diagnosis is possible earlier or at any time after birth, requiring only a simple blood sample. Genetic diagnosis is also important in cases where HCM develops later due to mutations associated with low penetrance and in those with hypertrophy caused by hypertension in young individuals, which are clinically indistinguishable. The genetic test identifies patients at risk of developing the disease before and regardless of the presence of symptoms or development of hypertrophy. The test also provides prognostic information, particularly the risk to SD. The need to determine individuals at high risk for subsequent HCM development and possible SD is greater in children, before hypertrophy develops. The ability to identify and separate individuals with high-risk mutations from those without mutations and, therefore, with no risk of developing the disease, provides further information for clinically treating these patients. Interventions, such as defibrillator implantation, should be indicated after stratifying the risk based upon clinical findings and genetics. Therefore, genetic tests of family members affected by HCM determine who is normal and who will probably develop the disease, as well as provide important prognostic data for asymptomatic children and individuals.

For the time being, genetic determination is experimental and performed only in few centers around the world, in addition to being a high-cost procedure. As it becomes viable, it will bring advantages to these patients and their families, and solution to some problems, such as what to do with a carrier of a malignant mutation, who is asymptomatic and can have, as the first symptom, SD? What should be done with a child who is a carrier of genetically diagnosed HCM and who has not yet developed hypertrophy?

These and other questions ought to be solved as time goes by. For the time being, new genes and new mutations are found on a daily basis, helping to elucidate the etiology of a disease that, until recently, was unknown and caused death in young people. 


\section{References}

1. Spirito P, Seidman CE, McKenna WJ, Maron BJ - The management of hypertrophic cardiomyopathy. N Engl J Med 1997; 33: 775-85.

2. Marian AJ, Roberts R - Recent advances in the molecular genetics of hypertrophic cardiomyopathy. Circulation 1995; 92: 1336-47.

3. Davies MJ, McKenna WJ - Hypertrophic cardiomyopathy: an introduction to pathology and pathogenesis. Br Heart J 1994; 72(suppl): S2-S3.

4. Marian AJ - Sudden cardiac death in patients with hypertrophic cardiomyopathy: from bench to bedside with an emphasis on genetic markers. Clin Cardiol 1995; 18: 189-98

5. Malik MS, Watkins $\mathrm{H}$ - The molecular genetics of hypertrophic cardiomiopathy. Curr Opinion Cardiol 1997; 12: 295-302.

6. Charron P, Dubourg O, Desnos M, et al - Genotype-phenotype correlations in familial hypertrophic cardiomyopathy. A comparison between mutations in the cardiac protein-C and the beta-myosin heavy chain genes. Eur Heart J 1998 19: $139-45$.

7. Watkins $\mathrm{H}$ - Multiple disease genes cause hypertrophic cardiomyopathy. $\mathrm{B}$ Heart J 1994; 72(suppl.): S4-S9.

8. Schwartz K, Carrier L, Guicheney P, Komadja M - Molecular basis of familial cardiomyopathies. Circulation 1995; 91: 532-40.

9. Watkins H, Rosenweig A, Hwang D, et al - Characteristics and prognostic implications of myosin missense mutations in familial hypertrophic cardiomyopathy. N Engl J Med 1992; 326: 1108-114.

10. Fananapazir L, Epstein ND - Genotype-phenotype correlations in hypertrophic cardiomyopathy: insights provided by comparisons of kindreds with distinct and identical $\beta$-myosin heavy chain gene mutations. Circulation 1994; 89: 22-32.

11. Watkins $\mathrm{H}, \mathrm{McKenna} \mathrm{WJ}$, Thierfelder $\mathrm{L}$, et al - Mutations in the genes for cardiac troponin $\mathrm{T}$ and a-tropomyosin in hypertrophic cardiomyopathy. N Engl J Med 1995; 332: 1058-64
12. Niimura H, Bachinski LL, Sangwatanaroj S, et al - Mutations in the gene for cardiac myosin-binding protein $\mathrm{C}$ and late-onset familial hypertrophic cardiomyopathy. N Engl J Med 1998; 338: 1248-57.

13. Flavigny J, Richard P, Isnard R et al - Identification of two novel mutations in the ventricular regulatory myosin light chain gene (MYL2) associated with familial and classical forms of hypertrophic cardiomyopathy. J Mol Med 1998; 76: $208-14$

14. Poetter K, Jiang H, Hassanzadeh S, et al - Mutations in either the essential or regulatory light chains of myosin are associated with a rare myopathy in human heart and skeletal muscles. Nat Genet 1995; 13: 63-9.

15. Lechin M, Quiñones MA, Omran A, et al - Angiotensin-I converting enzyme genotypes and left ventricular hypertrophy in patients with hypertrophic cardiomyopathy. Circulation 1995; 92: 1808-12.

16. Pfeufer A, Osterziel K-J, Urata $\mathrm{H}$ et al - Angiotensin-converting enzyme and heart chymase gene polymorphisms in hypertrophic cardiomyopathy. Am J Cardiol 1996; 78: 362-4.

17. Jan-Danser AH, Schalekamp MADH, Bax WA, et al - Angiotensin-converting enzyme in the human heart. Effect of the deletion/insertion polymorphism. Circulation 1995; 92: 1387-8

18. Iwai N, Ohmichi N, Nakamura Y, Kinoshita M - DD genotype of the angiotensinconverting enzyme gene is a risk factor for left ventricular hypertrophy. Circulation 1994; 90: 2622-8.

19. Ohishi M, Rakugi H, Ogihara T - Association between a deletion polymorphism of the angiotensin-converting-enzyme gene and left ventricular hypertrophy. N Engl J Med 1994; 331: 1097.

20. Ishanov A, Okamoto H, Yoneya K, et al - Angiotensinogen gene polymorphism in Japanese patients with hypertrophic cardiomyopathy. Am Heart J 1997; 133: 184-9. 